\title{
A Review of Routing Protocols for Practical Rural Wireless Mesh Networks (WMNs)
}

\author{
Ayomide Olanrewaju Ajayi \\ LANCASTER UNIVERSITY, \\ Lancaster, LA1 4WA, United \\ Kingdom
}

\author{
Adebisi Abimbola Adigun \\ LAUTECH, Ogbomoso \\ PMB 4000, Ogbomoso \\ Oyo-State, Nigeria
}

\author{
Wasiu Oladimeji Ismaila \\ LAUTECH, Ogbomoso \\ PMB 4000, Ogbomoso \\ Oyo-State, Nigeria
}

\begin{abstract}
The flexibility of WMNs affords their usage to provide broadband and communications services in many environments including rural domains. Routing protocols are central to the design of rural networks to ensure data availability and efficient performance especially under dynamic conditions of resource-constrained rural areas. In this paper, we review four routing protocols utilized for rural deployments namely AODV, OLSR, OSPF and BATMAN vis-à-vis the most critical metrics for rural requirements. Specifically, the comparative analysis shows the need for an objective evaluation of protocols for rural WMN scenarios. We also noted that metrics such as protocol overhead, convergence time and topology control remains critical for the performance of rural WMNs. Consequently, we argue that an objective performance evaluation offers a reliable selection criterion regarding the most efficient routing protocol when deploying WMNs. The study will further conduct simulation experiments to advocate the modification and synthesis of reliable protocols that will meet varying stringent requirements of remote settings.
\end{abstract}

\section{General Terms}

Routing protocols, Performance Efficiency, Wireless Mesh Networks.

\section{Keywords}

AODV, OLSR, OSPF, BATMAN, Objective Evaluation.

\section{INTRODUCTION}

Wireless mesh networks (WMNs) have viable economic potentials for the deployments of high-speed networks that are scalable and ubiquitous in a variety of environments [1]. WMNs are specialized wireless Ad hoc networks consisting of static mesh nodes or radios that communicate using intelligent ad hoc routing protocols. The use of routing enables capabilities for autonomous self-healing and route convergence under dynamic topologies when software glitches and hardware failures occur. The deployments of rural WMNs will remain indispensable due to their robustness and benefits in the eradication of the problems of the digital divide. Since Wireless Mesh Networks (WMNs) are variants of Mobile Adhoc NETworkS (MANETS); researchers adapts dynamic routing protocols (designed for MANETS) for utility in most WMN testbeds and deployments. The most fundamental choice relating to the option of routing is the mechanisms or procedures adopted. Routing mechanism describes algorithms that show how the protocols perform dynamic discovery and route selection. Three types exist namely distance vector, link-state, and hybrid routing protocols. In a distance vector (or reactive) routing protocol (e.g. AODV [2] and DSR [3]), all the routers send their routing tables (or a sub-set of its tables) to only their neighboring routers that periodically uses the received data to update their routing table on demand. Whereas, networks utilizing a link-state (or proactive) routing protocol (e.g. OLSR [4], BATMAN [5] and OSPF [6] allows each participating router to send their current state to all other routers subject to changes. The router uses this information to re-calculate the best path and to update its routing table accordingly. However, the scalability of these protocols for static WMNs is immensely challenging; hence, the use of hybrid protocols (such as HWMP) [7], which combines features of both reactive and proactive protocols to optimize routing efficiency. While reactive algorithms are simple techniques requiring minimal $\mathrm{CPU}$ resources; it reduces the protocol overhead at the expense of high latency. Whereas, proactive algorithms have high complexities requiring colossal CPU resources; it optimizes route selection based on a priori scheme, offers low latency but at the detriment of high protocol overhead and higher routing convergence.

While multi-hop WMNs remain an attractive solution for internet access; their performance efficiency, especially in rural domains, is partly dependent on effective routing protocols. However, the specifics of rural areas such as the use of inexpensive devices, link and device instability, wireless vulnerabilities, bandwidth limitations, power constraints and backhaul restrictions impair the performance. Moreover, the aforementioned affects both the data availability and network reliability, which are of prime concerns in remote domains. Therefore, the protocols utilized must ensure sensitivity to topology variations in order to optimize the utilization of the available bandwidth, minimize packet delivery delays (latency), reduce data access cost and ensure improved throughput under heterogeneous application/ services. Further, the choice of routing protocols for these settings needs to offer fast convergence, low overhead, minimal latency and jitter.

[8] investigate the survey of rural WMN deployments, which shows preferences for the usage of four (4) protocols for practical implementations namely ad hoc on demand vector (AODV), optimized link state routing (OLSR), open shortest path first (OSPF) and Better Approach To Mobile Ad hoc Networking (BATMAN). The selection criteria for these protocols have been subjective rather than an objective evaluation based on performance comparison or analysis. Also, we recognized the influence of factors such as ease and availability of implementations and performance results from other solitary investigations on the choice of these protocols. In order to evaluate the choice of the selection of the most efficient protocol given specific rural requirements, it becomes necessary to perform an objective comparative analysis under similar network parameters. The above serves as an optimal and appropriate guide for the selection of routing protocols in WMN deployments, which can fit varying domain requirements in the remote areas. 
In this paper, we offer a comparative analysis of routing protocols for rural WMN deployment case studies with great emphasis on the most critical performance metrics in resource-constrained domains. Furthermore, we establish the need for the objective evaluation of these routing protocols to determine the most efficient given specific network architectures, conditions, environmental interference and user applications under dynamic situations.

Specifically, we argue that metrics such as protocol overhead, topology control, protocol convergence, throughput, latency and packet delivery ratio are critical concerns in this context. The energy consideration remains critical due to the need for topology control and hence a fast routing convergence. Additionally, the work serves as a guide for the choice and selection of routing protocols tailored to the requirements of rural WMN implementations.

The organization of the remainder of this paper is as follows. Section 2 provides the basic overview of the WMN routing protocols under consideration as it relates to practical rural WMN deployments. Section 3 surveys existing work of performance evaluation of routing protocols for WMNNs. Section 4 presents that network model and metrics relevant to the rural domains. Section 5 offers brief discussion as well as conclude the paper with future investigations.

\section{ROUTING PROTOCOLS FOR RURAL WMN}

We present the apt descriptions of the following routing protocols employed for rural WNM deployments namely AODV, OLSR, OSPF and BATMAN.

\subsection{AODV}

The decentralized ad hoc on-demand distance vector (AODV) routing protocol is a reactive protocol designed for ad hoc networking with mobility support [2]. The algorithmic semantics combines features of both dynamic source routing (DSR), and destination sequenced and distance vector (DSDV) protocols to create and maintain route only when the need arises using a single path routing. It achieves its' routing philosophy using two fundamental procedural syntax, which are route discovery and route maintenance. AODV establishes route discovery in a similar fashion relating to the handshake communications mechanism; although it uses the trio of unicast, broadcast and multicast to flood route requests (RREQ), a node can only have one path towards a destination.

The AODV route discovery approach begins when a node requiring a valid link/path for packet traversal broadcasts route request (RREQ) information to the neighbors in search of a destination. Each of these neighbors also re-broadcast the RREQ to their neighbors and so on in a recursive manner until it floods the entire network topology with the RREQ. It establishes a route between a given source node and a destination (or intermediate) node that sends a unicast route reply (RREP) acknowledgment in the opposite unidirectional path to the source node. Route maintenance refers to the preservation of existing valid links or paths between a source node and the destination as much as required by the source node. This process depends on the life span of the source node that started the route request and the destination node. When a link breakage occurs due to mobility of the source node or power outage, route finding is once again started to establish another valid communication path. In contrast, if the failure affects the destination or any intermediate nodes, it propagates a route error message (RERR) message in the opposite direction to neighbors (and ultimately the source node) to inform them that the link is no longer active; route discovery cycle then begins again. It is good to also know that the AODV employs symmetric communications and so a request reply (RREP) takes the reverse path to that of the RREQ.

This AODV protocol is useful in scenarios where anticipated hostilities may persist like in typical generic rural setting, which lacks reliable power grid, subjected to harsh environmental conditions and remote due to the topography. The random network disconnections impact the mesh topology; generating a virtual mobility scenario even when nodes are static.

\subsection{OLSR}

The OLSR is a proactive routing protocol that models the operational semantics of link-state philosophy [4]. It performs the update of topological information periodically on each participating node. Unlike the AODV, it floods the entire network with topology messages for the information about the available route without the need to take cognizance of the network load and node mobility. To determine the optimality in terms of route selection, OLSR employs three (3) interdependent procedures namely neighbor sensing, message flooding and topology control. Neighbor Sensing helps any particular node to detect changes in the network topology via sensing of the network environment. This is achievable through the periodic HELLO messages sent by the nodes that receive acknowledgment based on the prevailing network configuration. The HELLO message offers the source address of the nodes sending the Hello packets as well as the information of all nodes that it senses. It also enables the determination of the link characteristics between the nodes and other nodes connected. Consequently, OLSR offers a scenario that allows every node to store information regarding all other connected nodes and well as the direction of existing routes that exist among them. However, this information stored on the nodes only subsists for a specified duration before the process of sensing commences again in a recursive manner. Therefore, OLSR uses periodic sensing to determine the steady-state of the network at any given period. Message flooding ensures that control traffic messages are sent to every node in the network Multipoint Relays (MPRs) to perform route selection and traffic forwarding. The MPRs are designate-nodes that forward broadcast messages that help to minimize the overhead of routing in comparison to other traditional flooding methods because of the re-transmission ability of selected MPRs. The computation of the optimal route between two destinations only requires the dissemination of partial link state information to determine the shortest path. More importantly, OLSR performs topology control (TC) by the periodic communication of TC messages among all network nodes by the MPRs, which are also only valid for a given time frame. The scalability potentials of OLSR motivate the preference in rural WMN deployments where fast convergence is desirable at the expense of incurring colossal computational overhead for control traffics.

\subsection{Batman}

In recent times, Better Approach To Mobile Ad hoc Networking (BATMAN) has emerged to be a choice of protocols in WMNs [5]. The protocol originates from the OLSR protocol; however, its' algorithmic semantic is different. Here, the in-degree of individual nodes determines the overall perception of the network. Each node perceives and maintains only the information about the best next-hop towards all other nodes via a selective flooding manner using originator messages (OGM). The OGMs is a quadruple (4tupule), which consist of the original sender's address, address 
of the node re-broadcasting the OGM, TTL (time to live) and the sequence number. The Batman nodes broadcast the OGMs periodically, and the recipients discard the OGM with lower sequences numbers that are now obsolete in the Batman procedural technique. The higher sequence numbers of the OGM indicates its freshness with respect to its life-span and helps to determine both route discovery and neighbor selection. Consequently, the global knowledge about the local topology becomes trivial and hence its performance enhancement and reliability over the OLSR by the reduction of signalling or routing overheads. BATMAN is suitably an alternative designed to offer enhanced performances over OLSR in terms of throughput, less delay, lower CPU load, significant reduction of routing overheads (low bandwidth utilization) and reduction in number of computations required by individual nodes. Therefore, the recent adoption of batman for next-generation rural wireless mesh networks seems imminent as seen in recent deployments based on the mesh potato routers in use for low-cost rural broadband connectivity and telephony. While BATMAN offers greater stability and high-packet delivery ratio (PDR) for telephone networks; the investigation of the performance comparison with other protocols is not exhaustive as reported in literatures. Specifically, there is a necessity to investigate the efficiency under similar network scenarios and parameters using simulation study and testbed analysis especially for the rural setting.

\subsection{OSPF}

The traditional OSPF routing protocol is also proactive linkstate routing protocol, which employs interval-driven exchange of control messages to achieve route discovery and topology maintenance [6]. The OSPF uses a multicast method to exchange "Hello" packets in order to establish bidirectional links between local neighbors. It further creates the shortest path trees using the shortest Path First (SPF) (via the Dijkstra's algorithm) for each route by sending link state advertisements (LSAs) to all routers. This allows the construction of a topology map of the entire network for each node in the network; a link state database (LSDB) results for usage for the calculating the routing table. It also has a mechanism for the provision of pair-wise synchronization of the LSDB between adjacent neighbors using additional control signaling such as database description messages and acknowledgments. OSPF has similar algorithmic semantics with OLSR being a link state protocol.

\section{RELATED SURVEY}

The performance evaluation studies regarding routing protocols presented here are not exhaustive. However, we examine a few investigations in this space as enumerated.

The study in [9] employs a real-life testbed to examine the performance comparison of BMX6 and OLSR routing protocols in terms of message overhead and convergence time. The analysis shows that the scalability of BMX6 is not significantly impacted when network node density and diameter increases compared to OLSR, where both can severely affect mesh scalability. The authors in [10] presents a simulation study of the multi-radio Ad-hoc On-demand Distance Vector (AODV-HP) routing protocol that allows differentiation amongst heterogeneous participating nodes in a hybrid wireless mesh network, where mobile mesh clients also participate in traffic routing and forwarding based on metrics such as packet delivery ratio, routing overhead and latency. The work also implements a route discovery mechanism that affords the optimal selection of interfaces in order to enhance the bandwidth capacity and reduce interference. The simulation results show that the variant protocol achieves significant performance improvement than the standard multi-radio AODV in all the metrics under high mobility and traffic load conditions.

The investigation in [11] and [12] are orthogonal and worthy of note. The work in [11] presents the NS2 simulation analysis of the performances of DSR, AODV, OLSR and DSDV protocols for wireless mesh backbone vis-a-vis the following metrics routing overhead, packet delivery ratio and end-to-end delay. Their simulation shows that DSR outperforms others in all scenarios under investigation. The performance of the AODV ranked second while that of DSDV is the least in this study. The study in [12] simulates the performance comparison of topology-based routing protocols such as DSDV, OLSR, AODV and DSR under a mesh implementation scenario. It provides an evaluation of these protocols based on the variation of network conditions such as network size, network load and node mobility. The results showed that for network size AODV performs better in the scenarios of high mobility and system load. OLSR performs much better than DSDV and DSR.

In addition, Johnson et al [13] evaluates the performance of BMXd routing protocol in comparison with the OLSR. The study measures the protocol overhead, throughput, CPU and memory consumption on an experimental 49-node indoor grid testbed. The study in [14] investigates the performance of OLSR, BATMAN and BABEL on a real-world testbed with a focus on the performance of multi-hop networks based on recovery from link failures. The results show that both BABEL and B.A.T.M.A.N outperform OLSR in terms of all performance metrics. The authors in [15] offer an experimental comparison of the performances of OLSR, Babel and BATMAN routing protocols. Their evaluation shows that the routing protocol overhead significantly determines the performance of both multi hop ad hoc networks.

Further studies in [16] investigates the use of quasi-fixed routing to address load balancing and fault tolerant problems in order to enhance the performances of wireless mesh networks. It further offers a discussion on how multiple gateways impacts the network performance. The study in [17] offers a comparative study of TORA and DSDV routing protocols particularly their performance trade-offs under various network conditions. The result shows that DSDV performs poorly under high mobility because it only provides a route per destination being an on-demand protocol, which significantly reduces the packet delivery ratio. In terms of the average end to end delay, DSDV and TORA have similar moderate performances. TORA utilizes an optimized method (table driven and on-demand routing) to reduce the protocol overhead, which enhances its performance over the DSDV that is only a table driven protocol. TORA performs better under high mobility scenarios than DSDV.

The study in [18] evaluates the performance of their proposed hybrid routing protocol for wireless mesh networks. The authors employ the DSDV and AODV respectively for nearby and remote destinations in this work. The hybrid routing protocol utilizes and maintains a shared routing table for their procedure. The proposed hybrid routing protocol exhibits a scalable performance that is better than DSDV but worse than AODV in terms of transmission delay. Whereas the performance was the reverse for the routing load as the number of nodes increases. The research in [19] offers the study of both the AODV and OLSR routing protocols for both wireless Ad-hoc and Mesh Network via simulation using 
OPNET modeler version. Specifically, their work evaluated the performances based on three metrics namely throughput, delay and network load for both networks. The AODV protocol performs better with static traffic because it requires less traffic overhead and bandwidth requirements. In contrast, the OLSR protocol shows greater efficiency in highly dense networks and sporadic traffic. They further found that the scalability of both the AODV and OLSR are limited flooding overhead and the size of the routing table (and topological updates messages) respectively.

The study in [20] is similar to the preceding work above. The authors utilized the OPNET simulator to model the performances of both DSR and AODV protocols under a wireless mesh network scenario. The simulation output shows the suitability of the AODV protocol for WMN especially under dynamic topologies while the DSR is not a reliable option for WMN implementations. Further, the work in [21], the authors examine the performance comparison of OLSR, OLSR-ETX, B.A.T.M.A.N and a variant of B.A.T.M.A.N protocol, called sw-B.A.T.M.A.N on a real-life WMN testbed with mobility support for mesh clients. The measurements from these experiments show that B.A.T.M.A.N-based approach is superior to the OLSR-based counterpart. The experimental evaluation further shows that the modified swB.A.T.M.A.N outperforms all other protocols for multimedia traffics.

The authors in [22] evaluate the performance characteristics of OLSR and AODV routing algorithms for Wireless Mesh Networks. Their work shows that the OLSR (Proactive routing) protocol outperforms the AODV (Reactive routing) in terms of the throughput capacity, whereas OLSR achieves higher packet delivery ratio than the AODV. In the overall, this simulation shows that proactive routing protocols have better performance ratios over the reactive protocols under multi-channel scenarios. Parveen Sharma [23] compares the performances of OLSR, AODV and ZRP based on three performance metrics namely throughput, latency and jitter. The author observed that change in data speed does not have any significant influence on the delay, whereas it does affect the throughput and jitter. This work concludes that for all node density variations within the range of 5-50; the AODV possess the best and optimal performance followed by the ZRP while the performances of OLSR is worst especially for lower node densities in small mesh networks.

Another recent work [24] evaluates the performances of the ADOV, DSR, GRP, OLSR and TORA protocols in order to determine the most efficient in terms of scalability, robustness, mobility, reliability and quality of service (QoS). The work further presents the variability of the throughput and delay metrics based on the three different ftp traffic loads. The study reveals that TORA has a leading high delay under both ftp medium load and ftp high load. GRP exhibits a somewhat stable (very short) delay under ftp high load and ftp low load. In the overall, OLSR significantly outperforms the others in terms of throughput under ftp medium load and ftp high load being a proactive (table-driven) protocol; TORA shows lead with high delay while GRP is the least efficient for all scenarios under consideration. Also, the authors in [25] investigates the performance analysis of ad hoc on demand distance vector (AODV), optimized link state routing (OLSR) and hybrid wireless mesh protocol (HWMP) in wireless mesh network scenario. Especially, it evaluates the effects of traffic loads, number of sources and network size on protocols utilized for the provision of rural broadband connectivity via simulation. HWMP exhibit performance enhancements by optimizing throughput and reduction of end to end delay than AODV and OLSR.

The study in [26] highlights the NS-2 simulation that measures the performance of DSR, AODV and DSDV protocols under a WMN scenario. The metrics under consideration includes hop count, packet delivery ratio (PDR), packet loss rate (PLR), routing communications overhead, throughput, Expected Transmission Count and Expected Transmission Time. The authors' analysis focuses the integration of techniques towards the synthesis of new protocols for WMNs. [27] investigates the performance of AODV and DSR using NS-2.34 simulation by evaluating metrics such as throughput, average end-end delay, PDR and NRL. Particularly, it evaluates these metrics for a configuration of a static backbone as well as under a random way point mobility model for client devices. The outcome shows that both AODV and DSR exhibit similar performance for packet delivery ratio and throughput under static configuration. However, the superiority of DSR compared to AODV under mobility considerations is proven for WMNs because it minimizes the routing overhead. This indicates that selecting DSR is appropriate when there is bandwidth scarcity.

\section{NETWORK PARAMETERS, CONDITIONS AND MODEL}

We propose a WMN configuration over different simulation environments and network parameters to investigate scalability performance. The nodes are configured considering the unique requirements in rural domains as shown in the rural WMN model architecture below.

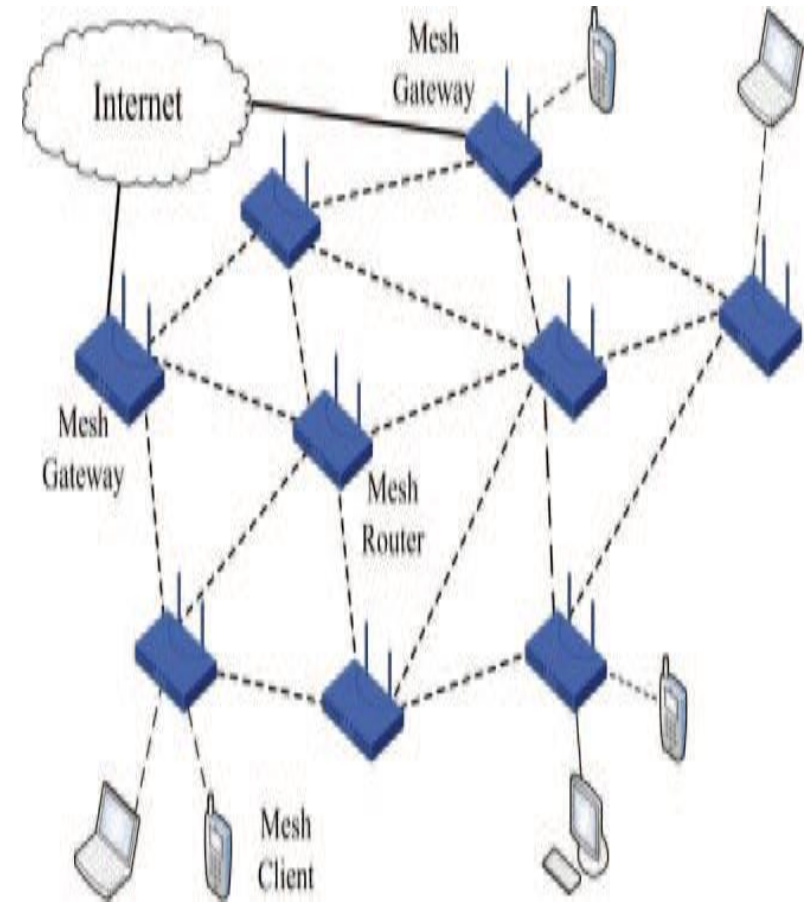

Figure 1. A Typical Rural WMN Configuration [28].

The critical metrics identified for evaluation of the efficiency of routing protocols includes topology control, communication overhead and convergence time. However, other metrics includes throughput, latency, packet delivery ratio (PDR) and jitter, some of which are more relevant when employing caching means to optimize the WMN gateway for 
improved data availability. We give brief descriptions of the relevance of the main metrics from a rural perspective.

\subsection{Topology Control}

Topology control (TC) describes how to sustain network connectivity under dynamic topologies. Topology control (TC) entails the ability to tune wireless link parameters [29] to reduce link interference, conserve radio energy and maintain network reliability [30] - [31]. Examples of such parameters include radio transmission power, bit rates and frequency domains. TC is affected by numerous factors including network size and node density. Under dynamic scenarios, TC protocol triggers changes to the route in networks in order to reduce the convergence time in nearly zero time [32] by reducing the time lag to adapt to changes in network topology. The benefit is that it helps to reduce jitter and increase the packet delivery ratio. In rural WMN architecture, efficient TC must support fast re-configuration of WMN components in both single and multiple gateway architectures must be nearly zero time. The time frame to support for fast re-configuration of WMN components in multiple gateway architecture must be nearly zero time.

\subsection{Routing Overhead}

Protocol overhead evaluates the associated cost for usage of network resources, traffic forwarding and maintenance. Routing overhead can affect the performance of both reactive and proactive protocols [33]. It is the Routing Overhead is the amount of generated control packets within the network. Especially, the significant impact is more severe in proactive protocols for large scale deployments [34]. Hence, the need to appropriately plan and reduce the broadcast interval for flooding control messages over the entire network becomes necessary due to bandwidth restrictions and power constraints in rural areas. The traffic usage, application scenarios, network size and node density, also affect the protocol overhead and hence, routing efficiency. The computational overhead adds to routing overhead, which impact routing efficiency due to excessive network flooding and congestion resulting from inadequate bandwidth. Limiting the overhead will improve routing and power (radio and node) efficiency and adopted caching techniques. Protocol overhead evaluates the associated cost for usage of network resources, traffic forwarding and maintenance. A minimal overhead is necessary due to due to bandwidth restrictions and energy constraints in rural locations.

\subsection{Routing Convergence}

Routing convergence [35] is a characteristic that defines the time required to re-establishing network connectivity after a topology change. Convergence represents the time frame or delay experienced by each node to learn the subsisting routes after network disruptions. It denotes the transition duration between a network failure and restoration (connectivity).

The convergence of routing protocols has significant effect on the efficiency of routing and network performance can be significant especially regarding data availability and network resiliency. A fast convergence in nearly zero-time is a metric that defines the fault-tolerance and resilience of the WMN in the advent of a node exclusion resulting from software glitches, hardware failure and link instability due to interference [36]. This is an important metric given the unique requirements of rural domains.

However, several other factors that impact the metrics above such as network size, traffic ccharacteristics, radio technology and internet gateway.

\section{DISCUSSION AND CONCLUSION}

\subsection{Review Analysis}

In [37] AODV has been shown to work well over a WMN with low traffic density; however, its scalability remains interactive as the load density increases. Furthermore, BATMAN has also been proven to have more reliable performance but has a small convergence time following topology variations [38]. In [5], the study affirms that OLSR possess a higher routing overhead than BATMAN due to the ability efficiently to administer route computation devoid of collection of unnecessary information about all the nodes in the network.

Also, [13] shows clear distinction in terms of the throughput of OLSR and BATMAN whereas another investigation reveals similarities of performance efficiency. In [39], the performance of OLSR is better than that of BATMAN especially under high mobility scenarios. [40] performs an experimental comparison of BATMAN and OLSR shows that BATMAN outperforms OLSR relative to throughput, access delay, CPU load and routing overhead unlike simulation that reveals similar performances. [41] demonstrates that OLSR outperforms the AODV in terms of network capacity and throughput. However, AODV has a better performance than OLSR concerning access latency. The OLSR maximizes the throughput as a result of the minimal communication overhead and low traffic load.

In addition, the simulation analysis in [42] demonstrates that B.A.T.M.A.N maintains stability under link variation and dynamic topologies. Whereas it exhibits low packet loss rate even in the face of wireless vulnerabilities such as thermal noise. Also, it has a low overhead threshold and experience a latency that is more acceptable than that of the OLSR. However, OLSR proves a reliable choice for high traffic load density and strong computational resources. Moreover, OLSR is energy-aware under high load density because it selects optimal path for the delivery of messages. The AODV remains ideal for moderate computational power and low traffic load. However, although it does not allow redundant overhead messages for inactive nodes, it exhibit indigent performance under increasing traffic loads due to lack of topology information. Here, we lay no further emphasis on OSPF because they are mainly of use in Autonomous System (AS).

The brief review shows that the diversities of various network scenario and use cases means that the direct application of a standard rule for the choice of routing is not the same for different conceptual views and deployment context. Consequently, an objective evaluation remains the most effective approach to choose an efficient routing protocol for any particular scenario.

\subsection{Conclusions}

The requirements of rural WMN deployments make the routing efficiency extremely challenging. The problems of wireless vulnerabilities, power failures, bandwidth limitations, VSAT communication constraints, and single gateway architecture exacerbates significant performance issues in the outlined rural context. Consequently, routing becomes problematic under dynamic topologies to improve the performance efficiency of these protocols for the rural WMN implementations requires an object evaluation of protocols used in these practical test-beds.

The optimization of the routing efficiency requires the adoption of metrics that fit the requirements of the settings. 
The topology control and convergence time for these protocols remains critical to the suitability and applicability and hence are factors for consideration.

Given the above, this work motivates the necessity to conduct an objective evaluation to investigate the performance of these protocols subject to metrics such as topology control, routing overhead and convergence time. This will further serve as an effective approach to ensure network reliability and availability while energy consumption can also be optimized.

An objective evaluation remains an effective approach to optimize the performance of rural WMNs in resourceconstrained settings. Further, it helps to ensure the optimization of nodal-energy in resource-constrained environments. The convergence of the routing protocol also determines their effectiveness under real-life situations.

Presently, an objective performance evaluation of AODV, OLSR, OSPF and BATMAN using simulation as well as reallife test-bed analysis for the critical metrics is ongoing.

\section{ACKNOWLEDGMENTS}

Our thanks goes to those who spared their time to review this work.

\section{REFERENCES}

[1] Akyildiz, Ian F., and Xudong Wang. "A survey on wireless mesh networks." Communications Magazine, IEEE 43, no. 9 (2005): S23-S30.

[2] Chakeres, Ian D., and Elizabeth M. Belding-Royer. "AODV routing protocol implementation design." In Distributed Computing Systems Workshops, 2004. Proceedings. 24th International Conference on, pp. 698703. IEEE, 2004.

[3] Johnson, D., Y. Hu, and D. Maltz. The dynamic source routing protocol (DSR) for mobile ad hoc networks for IPv4. RFC 4728, February, 2007.

[4] Clausen, Thomas, Philippe Jacquet, Cédric Adjih, Anis Laouiti, Pascale Minet, Paul Muhlethaler, Amir Qayyum, and Laurent Viennot. "Optimized link state routing protocol (OLSR)." (2003).

[5] D. Johnson, N. Ntlatlapa, and C. Aichele, "A pragmatic approach to mesh routing using batman," in 2nd IFIP International Symposium on Wireless Communications and Information Technology in Developing Countries, CSIR, Pretoria, South Africa, 6-7 October 2008, 2008.

[6] Moy, John T. OSPF complete implementation. Pearson Education, 2008.

[7] Yang, Kai, Jian-feng Ma, and Zi-hui Miao. "Hybrid routing protocol for wireless mesh network." In Computational Intelligence and Security, 2009. CIS'09. International Conference on, vol. 1, pp. 547-551. IEEE, 2009.

[8] Ajayi, Ayomide, Utz Roedig, Christopher Edwards, and Nicholas Race. "A survey of rural Wireless Mesh Network (WMN) deployments." In Wireless and Mobile, 2014 IEEE Asia Pacific Conference on, pp. 119-125. IEEE, 2014.

[9] Axel Neumann, Ester L'opez and Leandro Navarro; An evaluation of BMX6 for Community Wireless Networks.
[10] Asad Amir Pirzada and Marius Portmannt, High Performance AODV Routing Protocol for Hybrid Wireless Mesh Networks (2007)

[11] Ashraf, U.; Juanole, G.; Abdellatif, S.; , "Evaluating Routing Protocols for the Wireless Mesh Backbone", Wireless and Mobile Computing,Networking and Communications, 2007. WiMOB 2007. Third IEEE International Conference on , pp.40, 8-10 Oct. 2007

[12] Zakrzewska, A.; Koszalka, L.; Pozniak-Koszalka, I.; , "Performance Study of Routing Protocols for Wireless Mesh Networks," Systems Engineering, 2008. ICSENG '08. 19th International Conference on , vol., no., pp.331336, 19-21 Aug. 2008.

[13] Abolhasan, M.; Hagelstein, B.; Wang, J.C.-P.; , "Realworld performance of current proactive multi-hop mesh protocols," Communications, 2009. APCC 2009. 15th Asia-Pacific Conference on , vol., no., pp.44-47, 8-10 Oct. 2009

[14] Murray, D., Dixon, M.W. and Koziniec, T. (2010) An experimental comparison of routing protocols in multi hop ad hoc networks. In: Australasian Telecommunication Networks and Applications Conference, ATNAC, 31 October - 3 November, Auckland, New Zealand.

[15] Adward Lee and John beacli(2011), A Study of Routing Algorithms in Wireless Mesh Networks, British Journal of Science 1 September 2011, Vol. 1 (1)

[16] He, Q., Zhou, H., Wang, H. \& Zhu, L. (2007). Performance Comparison of Two Routing Protocols Based On WMN, International Conference on Wireless Communications, Networking and Mobile Computing, WiCom 2007, Page (s): 1726 - 1729.

[17] Oh, M. (2011). A Hybrid routing Protocol and Performance Evaluation in a wireless Mesh

[18] Kumar, S. and Sengupta, J. (2010). AODV and OLSR Routing Protocols for Wireless Ad-hoc and Mesh Networks, International Conference on Computer \& Communication Technology, IEEE, Pages 402-407.

[19] Yinpeng Yu, Yuhuai Peng, Lei Guo and Xingwei Wang, Performance Evaluation for Routing Protocols in Wireless Mesh Networks, 2010 International Conference on Educational and Information Technology (ICEIT 2010)

[20] Massimo Reineri, Roberto Rubino, Claudio Casetti and Carla-Fabiana Chiasserini, Experimental Performance Assessment of WMN Routing Protocols with Mobile Nodes, 2011

[21] Navtej Singh Sandhu, Navdeep Kaur Sandhu and Ashwinder Sing, Performance characteristics of OLSR and AODV protocols in Wireless Mesh Network, 2012

[22] Parveen Sharma, PERFORMANCE COMPARISON OF ROUTING PROTOCOLS IN WMNS, International Journal of Information Technology and Knowledge Management December 2012, Volume 6, No. 1, pp. 8388

[23] Armstrong Kadyamatimba and Zenzo Ncube, Analysis of Performance Evaluation in Routing Protocols of Wireless Mesh Networks, IJASCSE Volume 2, Special Issue 1, 2013 
[24] Zakaria, Azila, Hafizal Mohamad, Nordin Ramli, and Mahamod Ismail. "Performance evaluation of routing protocols in wireless mesh network." In Advanced Communication Technology (ICACT), 2013 15th International Conference on, pp. 1111-1115. IEEE, 2013.

[25] Rao, S. Siva Nageswara, YK Sundara Krishna, and K. Nageswara Rao. "Performance Evaluation of Routing Protocols in Wireless Mesh Networks." Performance Evaluation 68, no. 7 (2013).

[26] Bhushan, Shashi, Anil Saroliya, and Vijander Singh. "Implementation and Evaluation of Wireless Mesh Networks on MANET Routing Protocols." International Journal of Advanced Research in Computer and Communication Engineering Vol. 2, Issue 6, June 2013.

[27] A. Ephremides. Ad hoc networks: not an ad hoc field anymore. Wireless Communications and Mobile Computing, 2(5), 2002.

[28] M. Burkhart et al. Does Topology Control Reduce Interference? In Proc. ACM MobiHoc, 2004.

[29] E. Lloyd et al. Algorithmic Aspects of Topology Control Problems for Ad hoc Networks. In Proc. ACM MobiHoc, 2002.

[30] Liu and Bai: An overview of topology control mechanisms in multi-radio multi-channel wireless mesh networks. EURASIP Journal on Wireless Communications and Networking 2012 2012:324

[31] Zhu, Yun, Weirong Jiang, and Zhiming Zhang. "Routing Overhead Minimization in Large-Scale Wireless Mesh Networks."

[32] C. Adjih, E. Baccelli, and P. Jacquet, "Link State Routing In Wireless Ad-hoc Networks," Proc. of MILCOM 2003.

[33] Bilal Abdulhaq, Makhfudzah Binti Mokhtar and Aduwati Sali . Wireless Mesh Networks Protocols: State of the Art Review. International Journal of Advances in Computing and Information Technology, 2012, doi:10.6088/ijacit.12.15004
[34] E. Baccelli, F. Baker, M. Chandra, T. Henderson, J. Macker, R. White: Problem Statement for OSPF Extensions for Mobile Ad Hoc Routing. IETF InternetDraft, draft-baker-manet-ospfproblem-statement-00 (work in progress), 2003.

[35] Kaur, Navneet, and Jatinder Singh Saini. Performance enhancement of 802.11 based wireless mesh network by using Multi-Radio Multi-Channel." In Green Computing, Communication and Conservation of Energy (ICGCE), 2013 International Conference on, pp. 71-76. IEEE, 2013.

[36] Venkat Mohan.S and Kasiviswanath.N; Routing Protocols for Wireless Mesh Networks, International Journal of Scientific \& Engineering Research Volume 2, Issue 8, August-2011 1 ISSN 2229-5518 IJSER @) 2011 http://www .ijser.org

[37] Sikander Singh, Sukhwinder Singh, Dr Trilok Anand, Performance comparison of AODV, OLSR, OFLSR in Wireless Mesh Networks\| 2008.

[38] Rosario G. Garroppo, Stefano Giordano, Luca Tavanti, Experimental evaluation of two open source solutions for wireless mesh routing at layer-2\|, 2010.

[39] Sandhu, Davinder Singh, and Sukesha Sharma. "PERFORMANCE EVALUATION OF BATMAN, DSR, OLSR ROUTING PROTOCOLS-A REVIEW." Journal of Information \& Operations Management 3, no. 1 (2012).

[40] Sharma, Aastikta, and Narendran Rajagopalan. "A Comparative Study of BATMAN and OLSR Routing Protocols for MANETs."

[41] Jagdeep Singh and Rajiv Mahajan. "Performance Analysis Of AODV And OLSR Using OPNET" International Journal of Computer Trends and Technology (IJCTT) - volume 5 number 3-Nov 2013.

[42] Arda Yagci (2011), Comparison and Evaluation of Routing Mechanisms for Wi-Fi Mesh Networks, School of Computing, Blekinge Institute of Technology , Sweden. 